\title{
THE ASYMPTOTIC FORMS OF THE HERMITE AND WEBER FUNCTIONS*
}

\author{
BY \\ NATHAN SCHWID
}

1. Introduction. The classical Hermite equation,

$$
U^{\prime \prime}(z)-2 z U^{\prime}(z)+2 \kappa U(z)=0,
$$

which is satisfied by the Hermite polynomials

$$
U_{k}=(-1)^{k} e^{z^{2}} \frac{d^{k}\left(e^{-z^{2}}\right)}{d z^{k}}
$$

when the parameter $\kappa$ is a positive integer, has been widely discussed. The forms of its solutions, with respect to their asymptotic dependence upon $\alpha$, are of importance, and have been determined under certain restrictions upon the variables $z$ and $\kappa$. These restrictions, when heaviest, have confined $z$ to real and $\kappa$ to positive integral values; when lightest, they have permitted $z$ to vary in a strip of the complex plane of finite length and width, and $\kappa$ over the real axis. In the present paper it is purposed to remove these restrictions: to derive asymptotic forms of the solutions of the equation (1) valid in the entire $z$ plane for large values of $\kappa$, real or complex.

It may be recalled that the polynomials (2) were introduced into analysis by Hermite $\dagger$ in 1864 . Five years later, Weber $\ddagger$ noted that the harmonic functions applicable to the parabolic cylinder satisfy an ordinary differential equation of the form

$$
w^{\prime \prime}(z)+\left(2 \kappa+1-z^{2}\right) w(z)=0,
$$

which has since been generally known as the Weber equation. Whittaker§ showed in 1903 that this equation is obtainable from the Hermite equation (1) by a simple change of variable, and he determined the asymptotic expansion with respect to the real variable $z$, of a particular solution, the classic $D_{\kappa}(z)$.

\footnotetext{
* Presented to the Society, April 6, 1934; received by the editors May 17, 1934.

$\dagger$ Hermite, Sur un nouveau développement en série des fonctions, Comptes Rendus, vol. 58, pp. 93-100.

‡ Weber, Ueber die Integration der partiellen Differentialgleichung: $\quad \partial^{2} u / \partial x^{2}+\partial^{2} u / \partial y^{2}+k^{2} u=0$, Mathematische Annalen, vol. 1 (1869), pp. 1-36.

§ Whittaker, On the functions associated with the parabolic cylinder in harmonic analysis, Proceedings of the London Mathematical Society, vol. 35, pp. 417-427.
} 
Soon thereafter, Adamoff* obtained the asymptotic forms of the Hermite polynomials relative to the integral parameter $\kappa$, with the variable $z$ limited to real, finite values.

Watson $\dagger$ generalized these results. He developed the asymptotic expansion of $D_{k}(z)$ with respect to $z$ for all values of $\arg z$, and also determined, in a strip of the $z$ plane of finite width and length, the asymptotic forms of $D_{x}$ relative to the real parameter $\kappa$. His method, in the latter case, was a generalization of Adamoff's, whose procedure was based upon an elaborate transformation of a definite integral. More recently, Plancherel and Rotach $\ddagger$ derived asymptotic forms for the Hermite polynomials with respect to the integral parameter $\kappa$, for all real values of $z$. They used the saddle-point method $\S$ applied to a contour integral

$$
H_{\kappa-1}(x)=\frac{(\kappa-1) !}{(-1)^{\kappa-1}} \int_{C} \frac{e^{-z^{2} / 2-z x}}{z^{\kappa}} d z .
$$

This procedure, though a familiar one, is not so intimately connected with the differential equation.\|

In the present paper, the asymptotic forms of the solutions of the Hermite and Weber equations with respect to the complex parameter $\kappa$ are obtained for all complex values of $z$. This is done by utilizing formulas developed by Langer $\llbracket$ for the asymptotic solutions of an ordinary differential equation of the general structure

$$
w^{\prime \prime}(z)+p(z) w^{\prime}(z)+\left\{\rho^{2} \phi^{2}(z)+q(z)\right\} w(z)=0,
$$

where the parameter $\rho^{2}$ is large and the variable $z$ ranges over some region (finite or infinite) of the $z$ plane, in which the coefficient $\phi^{2}$ vanishes to some real non-negative power at one and only one point.

* Adamoff, Sur les expansions des polynomes $U_{n}=e^{\alpha x^{2} / 2} d^{n} e^{-\alpha x 2 / 2} / d x^{n}$ pour les grandes valeurs de $n$, Annales de l'Institut Polytechnique de St. Petersburg, 1906, pp. 127-143.

† Watson, G. N., The harmonic functions associated with the parabolic cylinder, Proceedings of the London Mathematical Society, (2), vol. 8, pp. 393-421.

$\ddagger$ Plancherel et Rotach, Sur les valeurs asymptotiques des polynomes d'Hermite, Commentarii Mathematici Helvetici, vol. 1 (1929), pp. 227-254.

$\S$ For a discussion of this method, see Courant-Hilbert, Methoden der Mathematischen Physik, vol. I, p. 435 .

|| Some additional related material of interest can be found in the following articles:

E. Hille, On the zeros of the functions of the parabolic cylinder, Arkiv för Matematik, Astronomi och Fysik, vol. 18 (1924), No. 26.

R. Nevanlinna, Über Riemannsche Flächen mit endlich vielen Windungspunkten, Acta Mathematica, vol. 58 (1932), pp. 295-373, especially pp. 344-355 and 361-372.

T Langer, R. E., On the asymptotic solutions of differential equations, etc., these Transactions, vo'. 34, No. 3, pp. 447-480. 
The asymptotic formulas here derived, which include as special cases the above mentioned forms of Watson (relative to large $\kappa$ ) and of Plancherel and Rotach, are shown to be in accord with the work of these men.

2. Preliminary considerations. The change of variable

$$
U=w e^{\varepsilon^{2} / 2}
$$

relates the equations (1) and (3), and in the latter, the substitutions

$$
z=(2 \kappa+1)^{1 / 2}(t+1), \rho=i(2 \kappa+1), w(z) \equiv u(t)
$$

further reduce the equation to the form

$$
u^{\prime \prime}(t)+\rho^{2}\left(t^{2}+2 t\right) u(t)=0 .
$$

This equation is of the type

$$
u^{\prime \prime}+\rho^{2} \phi^{2} u=0,
$$

for the solutions of which asymptotic formulas have been found by Langer.*

The equation (3) is unchanged when $z$ is replaced by $(-z)$, so that its principal solutions at the origin, which will be designated $w_{1}(z)$ and $w_{2}(z)$, are, respectively, even and odd functions. The variable $z$ may, accordingly, be restricted to some half plane, a convenient choice being

$$
\begin{gathered}
-\frac{\pi}{2}+\frac{1}{2} \arg (2 \kappa+1)<\arg z \leqq \frac{\pi}{2}+\frac{1}{2} \arg (2 \kappa+1), \text { that is, } \\
R(t) \geqq-1 .
\end{gathered}
$$

This part of the $t$ plane, cut along the negative real axis, so that

$$
-\pi<\arg t \leqq \pi,
$$

will be referred to as $R_{t}$, and the corresponding region (7) in the $z$ plane, cut from the origin to the point $z=(2 \kappa+1)^{1 / 2}$, will be referred to as $R_{z}$.

The function $\phi=\left(t^{2}+2 t\right)^{1 / 2}$ is evidently single-valued in $R_{t}$, and is completely specified if $\phi$ is chosen so that $\arg \phi=0$ when $\arg t=0$.

The complex parameter $\kappa$ is to be thought of as unbounded in magnitude but bounded from zero; its argument is restricted to the range of values

$$
-\frac{3 \pi}{2}<\arg (2 \kappa+1) \leqq \frac{\pi}{2} \text {, that is, }-\pi<\arg \rho \leqq \pi .
$$

The asymptotic forms given in $[\mathrm{L}] \dagger$ were obtained under certain hy-

* Langer considered the more general equation, $u^{\prime \prime}+\left(\rho^{2} \phi^{2}-\chi\right) u=0$, in which $\chi$ is analytic. In equation (6), $x \equiv 0$. Langer.

$\dagger$ This abbreviation will be used hereafter in referring to the previously mentioned paper of 
potheses on the coefficients of the differential equation, and a brief examination of these will suffice to show that they are satisfied by the equation (6).

(i) It is required that $\phi^{2}$ be of the structure $t^{\nu} \phi_{1}$, where $\nu \geqq 0$, and $\phi_{1}$ is single-valued, analytic, and non-vanishing in $R_{t}$; here this is obviously fulfilled, with $\phi^{2}=t(t+2)$.

(ii) The function $\Phi \equiv \int_{0}^{t} \phi(t) d t$, which, in this case, is of the specific form

$$
\Phi=\frac{1}{2}\left\{(t+1)\left(t^{2}+2 t\right)^{1 / 2}-\log \left[t+1+\left(t^{2}+2 t\right)^{1 / 2}\right]\right\},
$$

is to be non-vanishing in $R_{t}$ except at the origin.

That $\Phi$ as given in (10) fulfills this requirement is readily shown. In terms of the complex variable $\theta$ defined by the relation $t+1=\cos \theta$, the formula (10) can be written

$$
\Phi= \pm \frac{i}{4}(\sin 2 \theta-2 \theta) .
$$

From this relation it is easily verified that $\Phi$ vanishes only when $\theta=0$, i.e., when $t=0$.

Before examining the remaining hypotheses, it is desirable to discuss the map of the region $R_{t}$ upon the $\Phi$ plane. From the definition of $\Phi$, it is readily seen that the map is conformal, except at the origin, where the ratio of angles in $\Phi$ and $t$ planes is as $3: 2$. The manner in which the formula (10) determines the map is made apparent from the following table $\mathrm{I} ; R_{t}$ and its map $R_{\Phi}$ are shown in Figures 1 and 2 respectively.

TABLE I

\begin{tabular}{|c|c|c|}
\hline Line & $t$ & $\Phi$ \\
\hline$O A$ & 0 to $\infty$ & 0 to $\infty$ \\
\hline$O B$ & 0 to -1 & 0 to $-\frac{\pi i}{4}$ \\
\hline$B C$ & -1 to $-1+i \infty$ & $-\frac{\pi i}{4}$ to $-\infty-\frac{\pi i}{4}$ \\
\hline$O D$ & 0 to -1 & 0 to $\frac{\pi i}{4}$ \\
\hline$D E$ & -1 to $-1-i \infty$ & $\frac{\pi i}{4}$ to $-\infty+\frac{\pi i}{4}$ \\
\hline
\end{tabular}




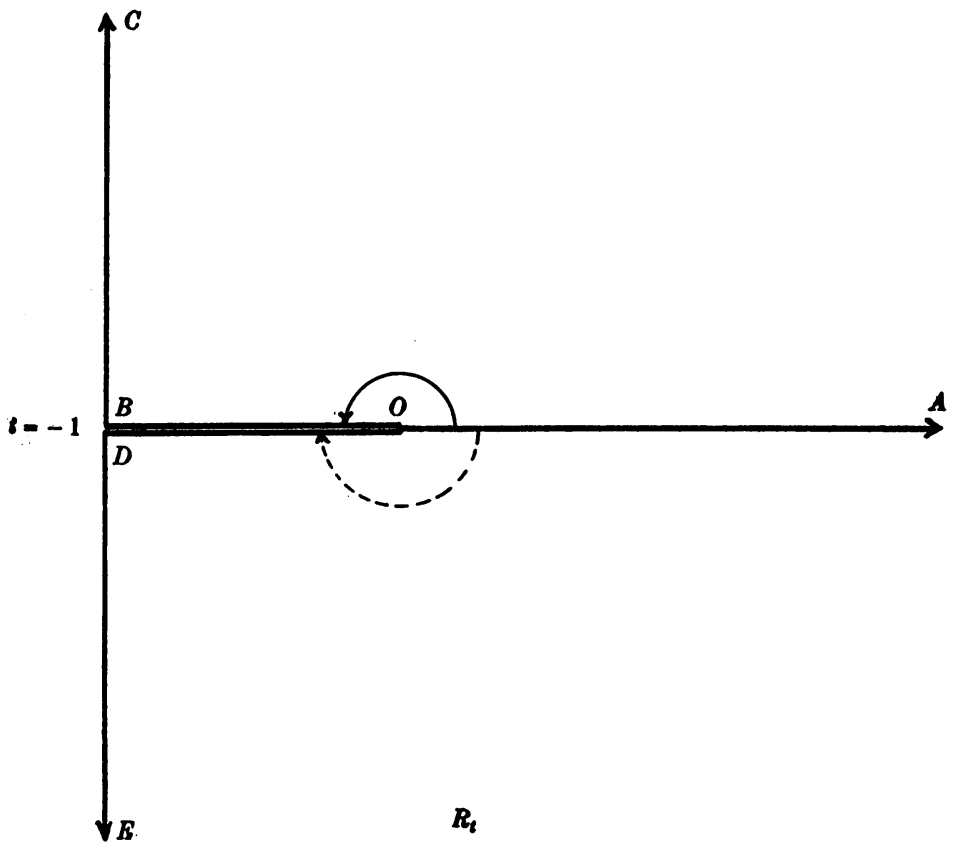

Figure 1

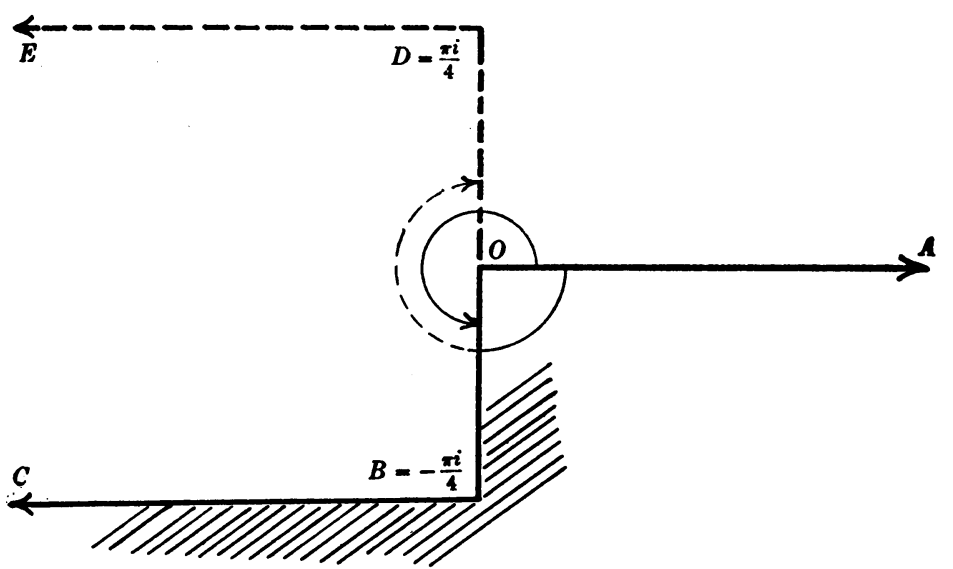

$\boldsymbol{R}_{\phi}$

FIGURE 2 
The consideration of the hypotheses may now be continued.

(iii) This, the hypothesis (iv) of [L], is a condition upon $R_{\xi}$, the map of $R_{t}$ upon the $\xi$ plane, where $\xi=\rho \Phi$. Without a consideration of details, it may be stated that, roughly, this hypothesis requires that the surface $R_{\xi}$ be one that contains in its interior only one segment of any horizontal line $\Im(\xi)=a$ constant. Since $R_{\xi}$ is obtained from $R_{\Phi}$ by a rotation about the origin and a magnification of the unit in the ratio $|2 \kappa+1|: 1$, it is evident from Figure 2 that the surface $R_{\xi}$ is such that the hypothesis is satisfied.

(iv) The region $R_{t}$ is to be such that for some positive number $M$, the relation

$$
\int\left|\frac{\Psi^{\prime \prime}}{\Psi \phi} d t\right|<M
$$

where $\Psi=\Phi^{1 / 6} / \phi^{1 / 2}$, is uniformly valid with respect to integrations over all arcs upon which $\Im(\xi)$ varies monotonically with $|\xi|$, and upon which $|t| \geqq N$, for some positive number $N$.

From the definition of $\Phi$ and the formula (10), it follows that $\Phi$ is of the structure

$$
\begin{aligned}
& \Phi=\frac{(2 t)^{3 / 2}}{3}[1+o(t)],|t|<2, \\
& \Phi=\frac{t^{2}}{2}\left[1+O\left(t^{-1}\right)\right],|t|>2,
\end{aligned}
$$

and by using the second of these relations, it may be readily verified that the integrand of (12) is $O\left(t^{-3}\right)$ when $|t|>2$. Hence, if $N$ is sufficiently large, the hypothesis is satisfied for all arcs of the required type.

The equation (6) is thus one to which the theorems proved in [L] are applicable.

3. The regions of validity. Let the regions $\Xi^{(h)}$ be defined by the relation

$$
(h-1) \pi+\epsilon \leqq \arg \xi \leqq(h+1) \pi-\epsilon \quad(h=0, \pm 1, \pm 2, \cdots),
$$

where $\epsilon$ is an arbitrary positive fixed constant sufficiently small. Asymptotic formulas, dependent upon $h$, are given in [L] for a certain set of independent solutions of the equation (6), and for any given $h$, the associated set of formulas is, by [Theorem $7, \mathrm{~L}$ ], valid in $\Xi^{(h)}$; i.e., valid for all values of $t$ for which $\xi(t)$ satisfies the relations (14). The particular values of the indices which will fix the regions for the present problem may be determined by noting that in $R_{\Phi}, \Phi$ is restricted to the values 
so that in $R_{\xi}$,

$$
-\frac{3 \pi}{2}<\arg \Phi \leqq \frac{3 \pi}{2}
$$

$$
\arg \rho-\frac{3 \pi}{2}<\arg \xi \leqq \arg \rho+\frac{3 \pi}{2}
$$

When $\kappa$ and its counterpart $\rho$ vary respectively in the half planes

$$
\begin{gathered}
-\frac{\pi}{2}<\arg (2 \kappa+1) \leqq \frac{\pi}{2}, \\
0<\arg \rho \leqq \pi,
\end{gathered}
$$

$\xi$ is restricted to the values

$$
-\frac{3 \pi}{2}<\arg \xi \leqq \frac{5 \pi}{2}
$$

this range comprises, wholly or in part, the regions $\Xi^{(h)}, h=-1,0,1,2$, with boundaries which may be conveniently specified in terms of $\Phi$ as follows:

$0<\arg \rho \leqq \pi:$

$$
\begin{aligned}
& \Xi^{(2)}: \quad \pi-\arg \rho+\epsilon \leqq \arg \Phi \leqq \frac{3 \pi}{2}, \\
& \Xi^{(1)}:-\quad-\arg \rho+\epsilon \leqq \arg \Phi \leqq 2 \pi-\arg \rho-\epsilon, \\
& \Xi^{(0)}:-\pi-\arg \rho+\epsilon \leqq \arg \Phi \leqq \pi-\arg \rho-\epsilon, \\
& \Xi^{(-1)}: \quad-\frac{3 \pi}{2} \leqq \arg \Phi \leqq-\arg \rho-\epsilon .
\end{aligned}
$$

Similarly, when $\kappa$ and $\rho$ are restricted to the respective half planes

$$
\begin{gathered}
-\frac{3 \pi}{2}<\arg (2 \kappa+1) \leqq-\frac{\pi}{2}, \\
-\pi<\arg \rho \leqq 0
\end{gathered}
$$

$\xi$ is confined by the bounds

$$
-\frac{5 \pi}{2}<\arg \xi \leqq \frac{3 \pi}{2}
$$

for which the corresponding regions are those with the indices $-2,-1,0,1$; these have the boundaries 
$-\pi<\arg \rho \leqq 0:$

$\Xi^{(-2)}: \quad-\frac{3 \pi}{2}<\arg \Phi \leqq-\pi-\arg \rho-\epsilon$,

$\Xi^{(-1)}: \quad-2 \pi-\arg \rho+\epsilon \leqq \arg \Phi \leqq-\arg \rho-\epsilon$,

(16)

$$
\begin{array}{ll}
\Xi^{(0)}: & -\pi-\arg \rho+\epsilon \leqq \arg \Phi \leqq \pi-\arg \rho-\epsilon, \\
\Xi(1): & -\arg \rho+\epsilon \leqq \arg \Phi \leqq \frac{3 \pi}{2} .
\end{array}
$$

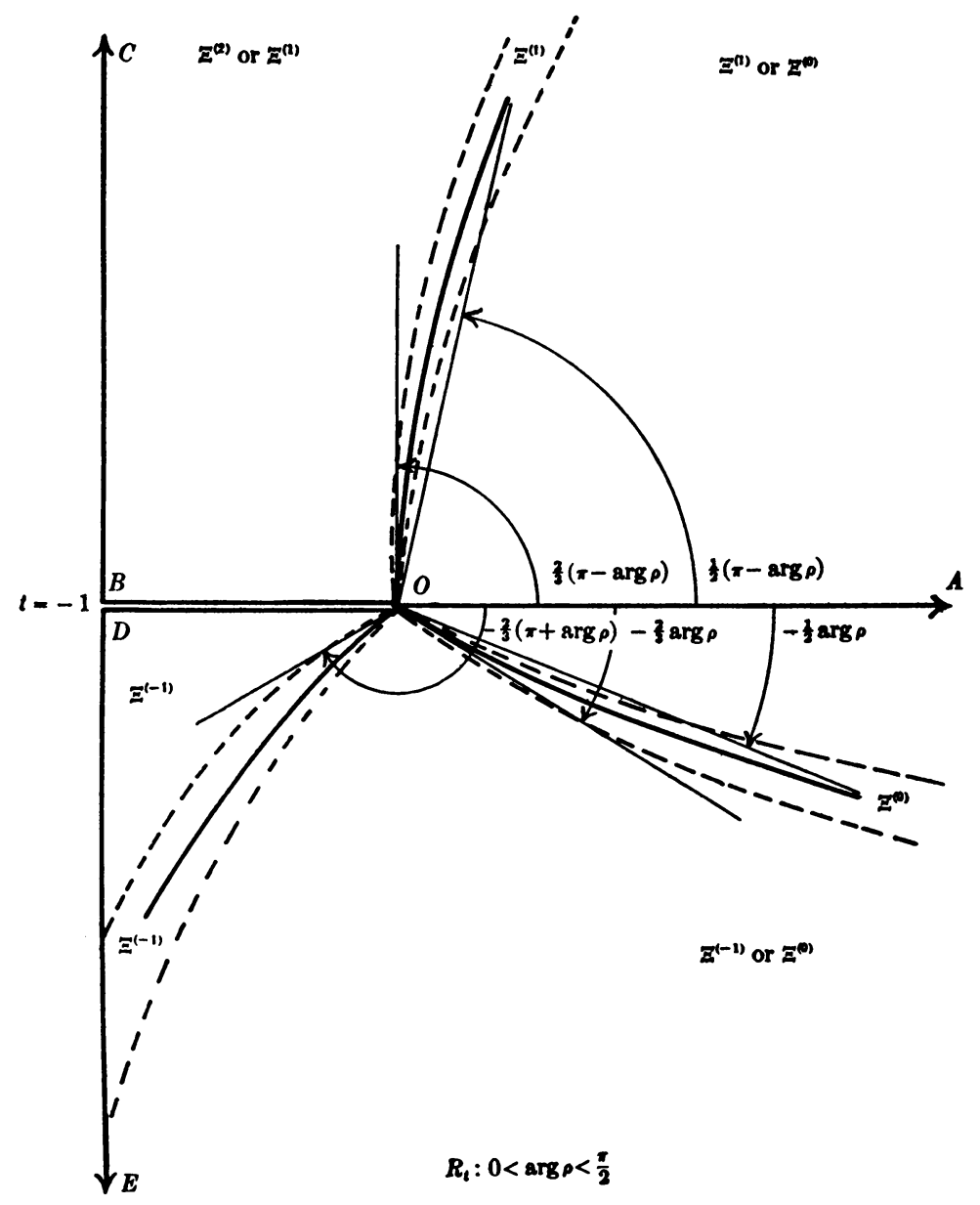

Figure 3(a) 


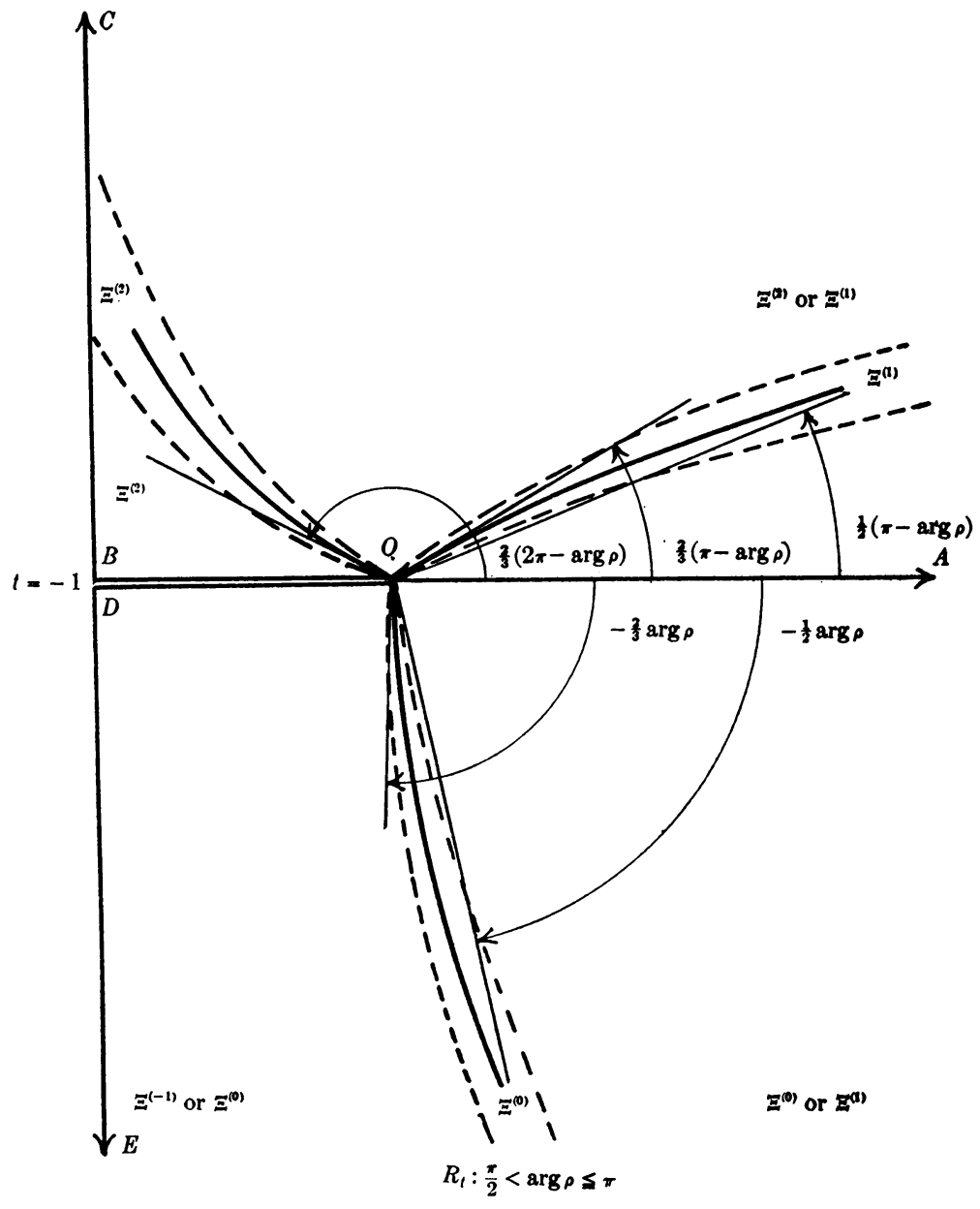

Figure 3(b)

The manner in which the boundary lines of (15) and (16) map as curves in $R_{t}$ becomes evident on examining the formulas (13); for from these it is seen that $\arg t \rightarrow \frac{1}{2} \arg \Phi$ when $t \rightarrow \infty$, and $\arg t \rightarrow \frac{2}{3} \arg \Phi$ when $t \rightarrow 0$. Hence a radial line arg $\Phi=\alpha$ maps as a curve in $R_{t}$ tangent at $t=0$ to the line $\arg t=2 \alpha / 3$, and asymptotic to the radial line $\arg t=\alpha / 2$.

The regions $\Xi^{(h)}$ when $\arg \rho>0$ are shown in Figure 3 for the typical cases $0<\arg \rho<\pi / 2, \pi / 2<\arg \rho \leqq \pi$, and the important special case, $\arg \rho=\pi / 2$, arg $\kappa=0$. To obtain the corresponding configurations when $\arg \rho<0$, it is only necessary to reflect Figure 3 in the axis of reals and to change the signs of the indices of the regions; these latter configurations, 
although not specifically given here, will, for convenience, be referred to as Figure 4 . The boundary curves in $R_{z}$ are readily obtained from Figures 3 and 4 by the appropriate change of unit, rotation and translation.

It will be noted that the regions overlap for consecutive values of $h$. A set of asymptotic formulas will be given for each region, and in the part common to two, either of the associated sets of formulas may be used, since they are asymptotically equivalent. Hence the exact location of the boundary curves of the regions (the dotted lines of Figure 3 ) is not important, for if at any point the validity of one form is in doubt, it will be certain that the other of the two formulas is then valid.

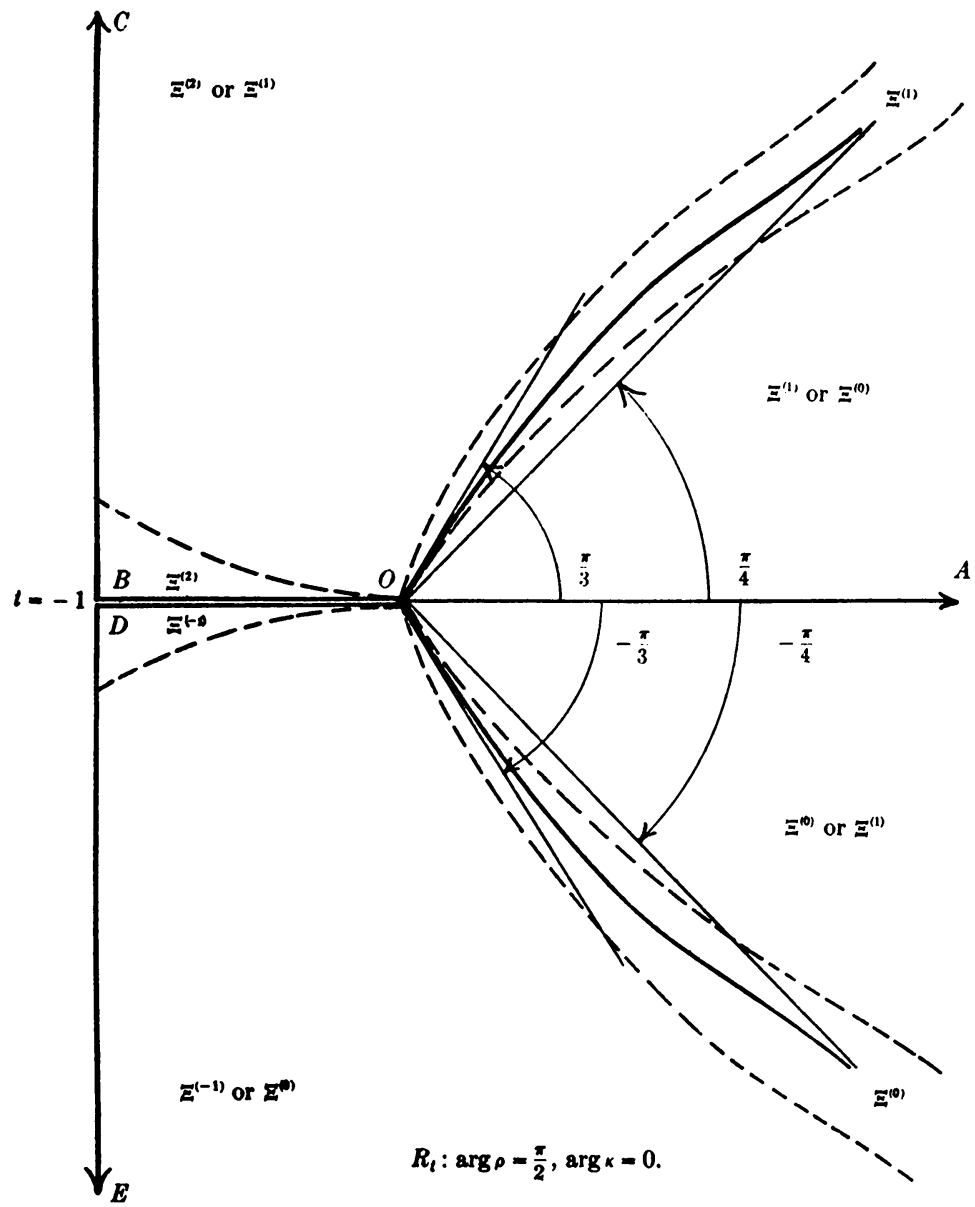

Figure 3(c) 
The forms derived in $\$ 4$ are subject to the restriction that $|\rho|$ and $|\xi|$ be large; subsequently, forms are derived for bounded values of $|\xi|,|\rho|$ large. From the structure of $\Phi$ as shown in equations (13), it is evident that $|\xi|$ is bounded when $t=O\left(|\rho|^{-2 / 3}\right)$, or, in terms of $z$, when $z=O\left(|\rho|^{1 / 2}\right)$. Hence the asymptotic forms for large $\xi$ are valid in the regions $\Xi^{(h)}$ as shown in Figures 3 and 4 with the exception of a region of dimension $O\left(|\rho|^{-2 / 3}\right)$ about the point $t=0$; i.e., about $z=(2 \kappa+1)^{1 / 2}$; in this latter region, the forms obtained for bounded values of $\xi$ apply.

Figure 4(a): $R_{t},-\pi / 2<\arg \rho \leqq 0$; this is Figure 3(a) reflected in real axis, and signs of indices changed.

Figure 4(b): $R_{t},-\pi<\arg \rho<-\pi / 2$; Figure 3(b) reflected in real axis, signs of indices changed.

Figure 4(c): $R_{t}$, arg $\rho=-\pi / 2$, arg $\kappa=-\pi$; Figure 3(c) reflected in real axis, signs of indices changed.

4. The asymptotic forms of the Weber functions. The solutions $w_{j}(z)$ of the equation (3) are expressible linearly in terms of any independent set of solutions, $u_{j}(t)$, of the equation (6), and these expressions, identities in $t$, may be written

$$
w_{j}(z) \equiv A_{1 j}^{(k)} u_{1}(t)+A_{2 j}^{(k)} u_{2}(t) \quad(j=1,2),
$$

in which the superscript $k$ has a significance which will presently be explained. The quantities $A_{l j}^{(k)}$ are constants with respect to $t$, but may depend upon $\rho$, so that any value of $t$ in $R_{t}$ may be used for their determination. The identities (17) reduce, when $t=-1$, to linear systems in $A_{l j}^{(k)}$ :

$$
\begin{aligned}
& A_{11}^{(k)} u_{1}(-1)+A_{21}^{(k)} u_{2}(-1)=1, \\
& A_{11}^{(k)} u_{1}^{\prime}(-1)+A_{21}^{(k)} u_{2}^{\prime}(-1)=0 ; \\
& A_{12}^{(k)} u_{1}(-1)+A_{22}^{(k)} u_{2}(-1)=0, \\
& A_{12}^{(k)} u_{1}^{\prime}(-1)+A_{22}^{(k)} u_{2}^{\prime}(-1)=(2 \kappa+1)^{1 / 2} .
\end{aligned}
$$

To solve these systems, the quantities $u_{j}(-1)$ and $u_{j}{ }^{\prime}(-1)$ must be computed. Asymptotic forms are given in [L] for an independent set of solutions of equation (6), which, together with their derivatives, assume at $t=0$ certain values.* These values are not, in themselves, germane to the present problem and are not here reproduced, but the functions $u_{j}$ of (17) are to be thought of as those solutions of (6) which take on at $t=0$ values as prescribed

* Cf. [L], p. 460, equations (23). 
in [L] and for which asymptotic formulas are there given.* Because of the presence of the Stokes' phenomenon, the asymptotic representation of a solution $u_{j}$ is different in distinct regions $\Xi^{(h)}$. Hence, in order to compute $u_{j}(-1)$ and $u_{j}{ }^{\prime}(-1)$ it is necessary to know in what region the variable $\xi$ is found when $t=-1$. Since

it follows that

$$
\xi(-1)=\frac{\pi \rho}{4} \exp \left(\frac{3 \pi i}{2}\right)
$$

$$
\begin{aligned}
& \xi(-1) \text { is in } \Xi^{(2)}, 0<\arg \rho \leqq \pi, \\
& \xi(-1) \text { is in } \Xi^{(1)},-\pi<\arg \rho \leqq 0,
\end{aligned}
$$

and the superscript $k$ of (17) will be assigned the values 2 or 1 according as $\rho$ is in its upper or lower half plane.

The quantities $u_{j}(-1)$ and $u_{j}{ }^{\prime}(-1)$ are now computed without difficulty from the formulas of $[\mathrm{L}]$, their values being $\ddagger \ddagger$

(19a)

$$
0<\arg \rho \leqq \pi \text { : }
$$

$$
u_{j}(-1)=e^{( \pm 1-1) \pi i / 3}\left(\frac{2}{\pi}\right)^{1 / 2}(2 \kappa+1)^{-1 / 6}\left[\cos \frac{\pi}{2}\left(\kappa \pm \frac{1}{3}\right)\right]
$$

$$
u_{j}^{\prime}(-1)=e^{( \pm 1-1) \pi i / 3}\left(\frac{2}{\pi}\right)^{1 / 2}(2 \kappa+1)^{5 / 6}\left[\sin \frac{\pi}{2}\left(\kappa \pm \frac{1}{3}\right)\right], j=1,2 ;
$$

$-\pi<\arg \rho \leqq 0:$

$$
\begin{aligned}
& u_{j}(-1)=e^{(7 \mp 2) \pi i / 6}\left(\frac{2}{\pi}\right)^{1 / 2}(2 \kappa+1)^{-1 / 6}\left[\sin \frac{\pi}{2}\left(\kappa \mp \frac{1}{3}\right)\right], \\
& u_{j}^{\prime}(-1)=e^{(\overline{1} \overline{+} 2) \pi i / 6}\left(\frac{2}{\pi}\right)^{1 / 2}(2 \kappa+1)^{5 / 6}\left[\cos \frac{\pi}{2}\left(\kappa \mp \frac{1}{3}\right)\right], j=1,2 .
\end{aligned}
$$

From these formulas, the determinant of the systems (18) is computed to be $(2 \kappa+1)^{2 / 3} e^{\pi i / 3} 3^{1 / 2}[1] / \pi$, and the systems are found to have the solutions

$$
A_{l 1}^{(2)}=e^{(1 \mp 1) \pi i / 3}\left(\frac{2 \pi}{3}\right)^{1 / 2}(2 \kappa+1)^{1 / 6}\left[\cos \frac{\pi}{2}\left(\kappa \pm \frac{2}{3}\right)\right]
$$

$$
A_{l 2}^{(2)}=e^{(1 \mp 1) \pi i / 3}\left(\frac{2 \pi}{3}\right)^{1 / 2}(2 \kappa+1)^{-1 / 3}\left[\sin \frac{\pi}{2}\left(\kappa \pm \frac{2}{3}\right)\right] \text {, }
$$

* Ibid., p. 462, formulas (29).

$\dagger$ Double signs used in connection with two indices indicate that the upper sign is to be used with the first, the lower sign with the second index.

$\ddagger$ The symbol $[Q]$ will always denote a series

$$
Q+\sum_{m=1}^{\infty} E_{i n} / \rho^{m}
$$

in which the $E_{m}$ are bounded functions of $\rho$ and $t$, or if $t$ is not present, of $\rho$ alone. 


$$
\begin{aligned}
& A_{l 1}^{(1)}=e^{( \pm 2-7) \pi i / 6}\left(\frac{2 \pi}{3}\right)^{1 / 2}(2 \kappa+1)^{1 / 6}\left[\sin \frac{\pi}{2}\left(\kappa \mp \frac{2}{3}\right)\right], \\
& A_{l 2}^{(1)}=e^{( \pm 2-1) \pi i / 6}\left(\frac{2 \pi}{3}\right)^{1 / 2}(2 \kappa+1)^{-1 / 3}\left[\cos \frac{\pi}{2}\left(\kappa \mp \frac{2}{3}\right)\right], \quad l=1,2 .
\end{aligned}
$$

The asymptotic forms of $u_{j}(t)$ when $|\xi|>N$ are given in [L] as

$$
u_{j}(t) \sim \rho^{-1 / 6} \phi^{-1 / 2}\left\{a_{j 1}^{(h)} e^{i \xi} S_{1 h}+a_{j 2}^{(h)} e^{-i \xi} S_{2 h}\right\}, \quad j=1,2,
$$

in which the quantities $S_{i n}$ are functions having the general structure $1+O\left(\rho^{-1}\right)+O\left(\xi^{-1}\right)$, the quantities $a_{j i}$ are constants which can be computed from formulas in [L, pp. 460-61], and the superscript $h$ denotes the region $\Xi(h)$ in which the formula is valid. The substitution of this and the formulas (20) in the identity (17) leads to the asymptotic forms of $w_{j}(z)$, and the results obtained are summarized in the following theorems:

TheOREM I. The asymptotic forms of the principal solutions $w_{j}(z)$ of the equation (3), valid, when $-\pi / 2<\arg (2 \kappa+1) \leqq \pi / 2$, in the regions $\Xi^{(h)}$ as shown in Figure 3, are given by the formula

(21) $w_{j}(z) \sim \frac{1}{2\left(z^{2} /(2 \kappa+1)-1\right)^{1 / 4}}\left\{B_{j 1}^{2 h} e^{i \xi}+B_{j 2}^{2 h} e^{-i \xi}\right\}(j=1,2 ; h=2,1,0,-1)$, in which the functions $\exp ( \pm i \xi)$ are of the specific form

$$
e^{ \pm i \xi}=\exp \left(\mp\left(z^{2}-2 \kappa-1\right)^{1 / 2} z / 2\right)\left\{\frac{z}{(2 \kappa+1)^{1 / 2}}+\left(\frac{z^{2}}{2 \kappa+1}-1\right)^{1 / 2}\right\}^{ \pm(2 \kappa+1) / 2}
$$

\begin{tabular}{|c|c|c|c|c|}
\hline$h$ & $B_{11}^{2 h}$ & $B_{12}^{2 h}$ & $B_{21}^{2 h}$ & $B_{22}^{2 h}$ \\
\hline 2 & $(-1)^{-\kappa / 2}[1]$ & $(-1)^{(x+1) / 2}[1]$ & $\frac{(-1)^{(1-\kappa) / 2}}{(2 \kappa+1)^{1 / 2}}[1]$ & $\frac{(-1)^{\kappa / 2}}{(2 \kappa+1)^{1 / 2}}[1]$ \\
\hline 1 & $(-1)^{-\kappa / 2}[1]$ & $-2\left[\sin \frac{\pi \kappa}{2}\right]$ & $\frac{(-1)^{(1-\kappa) / 2}}{(2 \kappa+1)^{1 / 2}}[1]$ & $\frac{2}{(2 \kappa+1)^{1 / 2}}\left[\cos \frac{\pi \kappa}{2}\right]$ \\
\hline 0 & $(-1)^{\times / 2}[1]$ & $-2\left[\sin \frac{\pi \kappa}{2}\right]$ & $\frac{(-1)^{(\kappa-1) / 2}}{(2 \kappa+1)^{1 / 2}}[1]$ & $\frac{2}{(2 \kappa+1)^{1 / 2}}\left[\cos \frac{\pi \kappa}{2}\right]$ \\
\hline-1 & $(-1)^{x / 2}[1]$ & $(-1)^{-(k+1) / 2}[1]$ & $\frac{(-1)^{(\kappa-1) / 2}}{(2 \kappa+1)^{1 / 2}}[1]$ & $\frac{(-1)^{\kappa / 2}}{(2 \kappa+1)^{1 / 2}}[1]$ \\
\hline
\end{tabular}

and the quantities $B_{j l}^{2 h}$ are functions of $\kappa$ whose values in the regions $\Xi^{(h)}$ are shown in the appended table II.

TABLE II 
THEOREM II. The asymptotic forms of the principal solutions $w_{j}(z)$ of the equation (3) valid, when $-3 \pi / 2<\arg (2 \kappa+1) \leqq-\pi / 2$, in the regions $\Xi^{(h)}$ as explained in Figure 4, are given by the formula

$$
\begin{aligned}
w_{j}(z) \sim \frac{1}{2\left(\frac{z^{2}}{2 \kappa+1}-1\right)^{1 / 4}}\left\{B_{j 1}^{1 h} e^{i \xi}+B_{j 2}^{1 h} e^{-i \xi}\right\} \\
\quad(j=1,2 ; h=1,0,-1,-2),
\end{aligned}
$$

in which the functions $\exp ( \pm i \xi)$ are described by the formula (22), and the quantities $B_{j l}^{1 h}$ have values in $\Xi^{(h)}$ as shown in the table III.

\begin{tabular}{|c|c|c|c|c|}
\hline$h$ & $B_{11}^{1 h}$ & $B_{12}^{1 h}$ & $B_{21}^{1 h}$ & $B_{22}^{1 h}$ \\
\hline 1 & $(-1)^{-\alpha / 2}[1]$ & $(-1)^{(\alpha+1) / 2}[1]$ & $\frac{(-1)^{(1-\kappa) / 2}}{(2 \kappa+1)^{1 / 2}}[1]$ & $\frac{(-1)^{\kappa / 2}}{(2 \kappa+1)^{1 / 2}}[1]$ \\
\hline 0 & $2\left[\cos \frac{\pi \kappa}{2}\right]$ & $(-1)^{(\alpha+1) / 2}[1]$ & $\frac{2\left[\sin \frac{\pi \kappa}{2}\right]}{(2 \kappa+1)^{1 / 2}}$ & $\frac{(-1)^{\kappa / 2}}{(2 \kappa+1)^{1 / 2}}[1]$ \\
\hline-1 & $2\left[\cos \frac{\pi \kappa}{2}\right]$ & $(-1)^{-(x+1) / 2}[1]$ & $\frac{2\left[\sin \frac{\pi \kappa}{2}\right]}{(2 \kappa+1)^{1 / 2}}$ & $\frac{(-1)^{-\kappa / 2}}{(2 \kappa+1)^{1 / 2}}[1]$ \\
\hline-2 & $(-1)^{\kappa / 2}[1]$ & $(-1)^{-(\alpha+1) / 2}[1]$ & $\frac{(-1)^{(\kappa-1) / 2[1]}}{(2 \kappa+1)^{1 / 2}}$ & $\frac{(-1)^{-\kappa / 2}[1]}{(2 \kappa+1)^{1 / 2}}$ \\
\hline
\end{tabular}

TABLE III

5. Special forms when $\kappa$ is real. When the parameter $\kappa$ is real, fairly simple forms of $w_{j}(z)$ are valid inside the semi-circle $|z|=|2 \kappa+1|^{1 / 2}$ contained in the half plane (7). When $\kappa$ is positive, (7) is the half plane

$$
R(z) \geqq 0,
$$

and the semi-circle is entirely within the regions $\Xi^{(h)}, h=2,-1$. Then, by Theorem I, the forms that apply are

$$
\begin{aligned}
w_{1}(z) \sim & \frac{1}{2\left(1-\frac{z^{2}}{2 \kappa+1}\right)^{1 / 4}}\left\{\exp \left(i\left(\xi \mp \frac{\pi \kappa}{2} \mp \frac{\pi}{4}\right)\right)[1]\right. \\
& \left.+\exp \left(-i\left(\xi \mp \frac{\pi \kappa}{2} \mp \frac{\pi}{4}\right)\right)[1]\right\}
\end{aligned}
$$




$$
\begin{aligned}
w_{2}(z) \sim & \frac{1}{2(2 \kappa+1)^{1 / 2}\left(1-\frac{z^{2}}{2 \kappa+1}\right)^{1 / 4}}\left\{\exp \left(i\left(\xi \mp \frac{\pi \kappa}{2} \pm \frac{\pi}{4}\right)\right)[1]\right. \\
& \left.+\exp \left(-i\left(\xi \mp \frac{\pi \kappa}{2} \pm \frac{\pi}{4}\right)\right)[1]\right\}
\end{aligned}
$$

in which

$$
\arg \left(1-\frac{z^{2}}{2 \kappa+1}\right) \leqq 0 \text { when } y \geqq 0, \arg \left(1-\frac{z^{2}}{2 \kappa+1}\right)>0, y<0 .
$$

Attention has already been called (equation (11)) to the fact that with the substitution

$$
\cos \theta=\frac{z}{(2 \kappa+1)^{1 / 2}}
$$

$\xi$ takes the form

$$
\xi= \pm \frac{2 \kappa+1}{2}(\theta-\sin \theta \cos \theta)
$$

and if the sign of $\arg \left(1-z^{2} /(2 \kappa+1)\right)$ be determined as in (24), then here the upper sign applies when $y \geqq 0$ inside the semi-circle, the lower when $y<0$.

The variable $\theta$, inside the semi-circle, is of the structure

$$
\theta=\frac{\pi}{2}-\frac{z}{(2 \kappa+1)^{1 / 2}}+O\left(\kappa^{-3 / 2}\right)
$$

so that by (25), $\xi$ may be written

$$
\xi= \pm\left\{(2 \kappa+1) \frac{\pi}{4}-z(2 \kappa+1)^{1 / 2}+O\left(\kappa^{-1 / 2}\right)\right\} .
$$

The use of this formula in (24) gives to the latter the forms

$$
\begin{aligned}
& w_{1}(z) \sim \frac{1}{\left(1-\frac{z^{2}}{2 \kappa+1}\right)^{1 / 4}}\left\{\cos z(2 \kappa+1)^{1 / 2}+O\left(\kappa^{-1 / 2}\right)\right\}, \\
& w_{2}(z) \sim \frac{1}{(2 \kappa+1)^{1 / 2}\left(1-\frac{z^{2}}{2 \kappa+1}\right)}\left\{\sin z(2 \kappa+1)^{1 / 2}+O\left(\kappa^{-1 / 2}\right)\right\}, \\
& 0 \leqq|y| \leqq \epsilon,
\end{aligned}
$$

$\epsilon$ being an arbitrary fixed positive quantity sufficiently small. 
When $y>\epsilon$, the first of forms (24) may be written, with the aid of (26), in the form

$$
w_{1}(z) \sim \frac{\cos z(2 \kappa+1)^{1 / 2}}{\left(1-\frac{z^{2}}{2 \kappa+1}\right)^{1 / 4}}\left\{1+\frac{E_{1}+E_{2} \exp \left(2 i z(2 \kappa+1)^{1 / 2}\right)}{1+\exp \left(2 i z(2 \kappa+1)^{1 / 2}\right)} \kappa^{-1 / 2}\right\},
$$

in which $E_{j}$ are bounded functions of $z$ and $\kappa$. It follows that the numerator of the fraction inside the brace is also bounded in $z$ and $\kappa$. The denominator of this fraction vanishes only on the real axis, at the points

$$
x=\frac{2 n+1}{(2 \kappa+1)^{1 / 2}} \frac{\pi}{2} \quad(n=0,1,2, \cdots)
$$

so that the denominator is non-vanishing if $y>\epsilon$; but more than this, it is then bounded from zero, uniformly in $z$ and $\kappa$; for if $2 \kappa+1>M^{2}$, then

$$
\begin{aligned}
&\left|1+\exp \left(2 i z(2 \kappa+1)^{1 / 2}\right)\right|= \mid 1-\exp \left(-2 y(2 \kappa+1)^{1 / 2}\right)\left(\exp \left(-2 y(2 \kappa+1)^{1 / 2}\right)\right. \\
&\left.+2 \cos x(2 \kappa+1)^{1 / 2}\right) \mid \\
&\left|1+\exp \left(2 i z(2 \kappa+1)^{1 / 2}\right)\right| \geqq 1-\exp \left(-2 y(2 \kappa+1)^{1 / 2}\right)>1-e^{-2 \epsilon M}>0
\end{aligned}
$$

hence $w_{1}(z)$ may be written, when $y>\epsilon$,

$$
w_{1}(z) \sim \frac{\cos z(2 \kappa+1)^{1 / 2}}{\left(1-\frac{z^{2}}{2 \kappa+1}\right)^{1 / 4}}\left\{1+O\left(\kappa^{-1 / 2}\right)\right\} .
$$

The same conclusion is reached when $|y|>\epsilon$, and similar results hold for $w_{2}(z)$.

When $\kappa$ is real and negative, the half plane (7) is described by the relation

$$
\Im(z) \leqq 0,
$$

and in the regions $\Xi^{(h)}, h=1,-2$, the forms that apply are those given in (24). Inside the semi-circle under consideration, the results that obtain are similar to those obtained when $\kappa>0$. The facts developed in this section are summed up in the following theorems:

Theorem III. When the parameter $\kappa$ is positive, the asymptotic forms of $w_{j}(z)$ inside the semi-circle $|z|=(2 \kappa+1)^{1 / 2}$ contained in the half plane $R(z) \geqq 0$ are given by the formulas

$$
w_{1}(z) \sim \frac{1}{\left(1-\frac{z^{2}}{2 \kappa+1}\right)^{1 / 4}}\left\{\begin{array}{l}
\cos z(2 \kappa+1)^{1 / 2}+O\left(\kappa^{-1 / 2}\right), 0 \leqq|y| \leqq \epsilon, \\
\cos z(2 \kappa+1)^{1 / 2}\left(1+O\left(\kappa^{-1 / 2}\right)\right),|y|>\epsilon,
\end{array}\right.
$$


(27)

$$
w_{2}(z) \sim \frac{1}{(2 \kappa+1)^{1 / 2}\left(1-\frac{z^{2}}{2 \kappa+1}\right)^{1 / 4}}\left\{\begin{array}{r}
\sin z(2 \kappa+1)^{1 / 2}+O\left(\kappa^{-1 / 2}\right), \\
0 \leqq|y| \leqq \epsilon, \\
\sin z(2 \kappa+1)^{1 / 2}\left(1+O\left(\kappa^{-1 / 2}\right)\right), \\
|y|>\epsilon,
\end{array}\right.
$$

in which

$$
\arg \left(1-z^{2} /(2 \kappa+1)\right)<0 \text { when } y \geqq 0,
$$

and $\epsilon$ is an arbitrary fixed positive quantity, sufficiently small.

THEOREM IV. When the parameter $\kappa$ is negative, the asymptotic forms of $w_{i}(z)$ inside the semi-circle $|z|=\left.|2 \kappa+1|\right|^{1 / 2}$ contained in the half plane $\Im(z) \leqq 0$ are given by formulas (27), in which all restrictions on $y$ are replaced by corresponding ones upon $x$.

6. The forms for bounded values of $\xi$. The variable $\xi$ is bounded in a region of $O\left(|\kappa|^{-2 / 3}\right)$ about the origin of $R_{t}$, and here, as has been previously pointed out, the asymptotic forms of Theorems I to IV are not valid. For this range of values, the solutions $u_{j}(t)$ are described by special asymptotic formulas which are given in [L] as

$$
u_{j}(t)=\Psi \xi^{1 / 3} J_{\mp_{1 / 3}}(\xi)+E(t, \kappa) / \kappa^{4 / 3}, \quad j=1,2,
$$

in which $E$ is a bounded function, and $J_{\alpha}$ is the familiar symbol for the Bessel function of the first kind of order $\alpha$. The function $\Psi=\Phi^{1 / 6} / \phi^{1 / 2}$, it may be noted, is analytic, single-valued, and non-vanishing in the neighborhood of $t=0$. The substitution of (28) into the identity (17) leads to asymptotic forms for $w_{j}(z)$ in which, by virtue of the fact that $t=O\left(|\kappa|^{-2 / 3}\right)$ in the region under discussion, approximations for the functions $\phi, \Phi$ and $\xi$ readily obtainable from (13) may be used. The facts are embodied in the following theorem:

THEOREM V. The asymptotic forms of $w_{i}(z)$ when $|\xi|$ is bounded are given by the formula

$$
\begin{array}{r}
(29) w_{j}(z)=\left(\frac{2 \pi \Phi}{3 \phi}\right)^{1 / 2} e^{\pi / 6}(2 \kappa+1)^{1 / 2}\left\{C_{j 1}^{(k)} J_{-1 / 3}(\xi)+C_{j 2}^{(k)} J_{1 / 3}(\xi)+\frac{E(t, \kappa)}{\kappa}\right\} \\
(j=1,2 ; k=2,1),
\end{array}
$$

in which $\arg \rho>0$ when $k=2$, arg $\rho \leqq 0, k=1$, the approximations

$$
\begin{aligned}
\left(\frac{\Phi}{\phi}\right)^{1 / 2} & \sim\left(\frac{2}{3}\right)^{1 / 2}\left\{\frac{z}{(2 \kappa+1)^{1 / 2}}-1\right\}^{1 / 2}, \\
\xi & \sim \frac{i(2 \kappa+1) 2^{3 / 2}}{3}\left\{\frac{z}{(2 \kappa+1)^{1 / 2}}-1\right\}^{3 / 2}
\end{aligned}
$$

may be used, and the coefficients $C_{j l}^{(k)}$ have the values shown in the table IV. 
TABLE IV

\begin{tabular}{|c|c|c|c|c|}
\hline$k$ & $C_{11}^{(k)}$ & $C_{12}^{(k)}$ & $C_{21}^{(k)}$ & $C_{22}^{(k)}$ \\
\hline 2 & {$\left[\cos \frac{\pi}{2}\left(x+\frac{2}{3}\right)\right]$} & $e^{2 \pi i / 3}\left[\cos \frac{\pi}{2}\left(\kappa-\frac{2}{3}\right)\right]$ & $\frac{\left[\sin \frac{\pi}{2}\left(\kappa+\frac{\pi}{3}\right)\right]}{(2 \kappa+1)^{1 / 2}}$ & $\frac{e^{2 \pi i / 3}\left[\sin \frac{\pi}{2}\left(\kappa-\frac{\pi}{3}\right)\right]}{(2 \kappa+1)^{1 / 2}}$ \\
\hline 1 & $e^{-6 \pi / 1 / 0}\left[\sin \frac{\pi}{2}\left(x-\frac{2}{3}\right)\right]$ & $i\left[\sin \frac{\pi}{2}\left(\kappa+\frac{2}{3}\right)\right]$ & $\frac{e^{\pi / 6}\left[\cos \frac{\pi}{2}\left(\kappa-\frac{\pi}{3}\right)\right]}{(2 \kappa+1)^{1 / 2}}$ & $\frac{-i\left[\cos \frac{\pi}{2}\left(\kappa+\frac{\pi}{3}\right)\right]}{(2 \kappa+1)^{1 / 2}}$ \\
\hline
\end{tabular}

When $\xi$ is small, formula (29) may be used to compute series which represent asymptotically the solutions $w_{j}$ in the neighborhood of $z=(2 \kappa+1)^{1 / 2}$. It is convenient here to introduce the variable $\eta$, defined by the relation

$$
\eta=\frac{z^{2}-2 \kappa-1}{z^{2 / 3}}
$$

and since $z^{2}-2 \kappa-1=o\left(|\kappa|^{1 / 3}\right)$, when $\xi$ is small, $\eta=o(1)$. Leading terms of the power series in $\eta$ for the functions $\phi, \xi$ and $\psi$ are readily computed, and are found to be the following:

$$
\begin{aligned}
\phi & =\frac{\eta^{1 / 2}}{z^{2 / 3}}\left(1+\frac{\eta}{2 z^{4 / 3}}+\cdots\right), \\
\xi & =\frac{i}{3} \eta^{3 / 2}\left(1+\frac{\eta}{5 z^{4 / 3}}+\cdots\right), \\
\frac{\Psi}{\rho^{1 / 3}} & =\frac{1}{e^{\pi i / 6} 3^{1 / 6} z^{2 / 3}}\left(1+\frac{17}{60 z^{4 / 3}} \eta+\cdots\right) .
\end{aligned}
$$

Since $\xi$ is small, the quantities $\xi^{1 / 3} J_{\mp 1 / 3}(\xi)$ are essentially given by the leading terms of their power series expansions,

$$
\begin{aligned}
\xi^{1 / 3} J_{-1 / 3}(\xi) & =\frac{2^{1 / 3}}{\Gamma\left(\frac{2}{3}\right)}-\frac{\xi^{2}}{2^{5 / 3} \Gamma\left(\frac{5}{8}\right)}+\cdots, \\
\xi^{1 / 3} J_{1 / 3}(\xi) & =\frac{\xi^{2 / 3}}{2^{1 / 3} \Gamma\left(\frac{4}{3}\right)}+\cdots,
\end{aligned}
$$

and these, with the use of (32) and the familiar formula

$$
3^{1 / 2} \Gamma\left(\frac{1}{3}\right) \Gamma\left(\frac{2}{3}\right)=2 \pi,
$$

are expressible in terms of $\eta$ as follows: 


$$
\begin{gathered}
\xi^{1 / 3} J_{-1 / 3}(\xi)=\frac{6^{1 / 3} 3^{1 / 6} \Gamma\left(\frac{1}{3}\right)}{2 \pi}\left(1+\frac{\eta^{3}}{24}+\cdots\right), \\
\xi^{1 / 3} J_{1 / 3}(\xi)=\frac{e^{\pi i / 3} 6^{2 / 3} 3^{1 / 6} \Gamma\left(\frac{2}{3}\right)}{4 \pi} \eta+\cdots
\end{gathered}
$$

The quantities $(\Phi / \phi)^{1 / 2} J_{\mp 1 / 3}(\xi)$ found in (29) may also be written $\Psi(\xi / \rho)^{1 / 3} J_{\mp 1 / 3}(\xi)$; hence the leading terms of the expansions of these quantities in powers of $\eta$ are readily determined from (32) and (33), and the asymptotic forms of $w_{j}(z)$ for small values of $\xi$ are then at hand from the formula (29). The following theorem embodies the results:

THEOREM VI. The asymptotic forms of $w_{j}(z)$ when $|\xi|$ is small are given by the series

$$
\begin{aligned}
w_{j}(z)=\frac{(2 \kappa+1)^{1 / 2}}{6^{1 / 6} \pi^{1 / 2} z^{2 / 3}}\left\{D_{j 1}^{(k)} \Gamma\left(\frac{1}{3}\right)+D_{j 2}^{(k)} \frac{6^{1 / 3} \Gamma\left(\frac{2}{3}\right)}{2} \eta\right. \\
\left.+D_{j 1}^{(k)} \frac{\Gamma\left(\frac{1}{3}\right)}{24} \eta^{3}+\cdots+\frac{E(z, \kappa)}{\kappa}\right\} \quad(j=1,2 ; k=2,1),
\end{aligned}
$$

in which $k=2$ when $-\pi / 2<\arg (2 \kappa+1) \leqq \pi / 2, k=1$, when $-3 \pi / 2<\arg (2 \kappa+1)$ $\leqq-\pi / 2, \eta$ is defined by (31), $E$ is a bounded function, and the coefficients $D$ are given in the table $\mathrm{V}$ :

TABLE V

\begin{tabular}{|c|c|c|c|c|}
\hline$k$ & $D_{11}^{(k)}$ & $D_{12}^{(k)}$ & $D_{21}^{(k)}$ & $D_{22}^{(k)}$ \\
\hline 2 & $\cos \frac{\pi}{2}\left(\kappa+\frac{2}{3}\right)$ & $-\cos \frac{\pi}{2}\left(\kappa-\frac{2}{3}\right)$ & $\frac{\sin \frac{\pi}{2}\left(\kappa+\frac{2}{3}\right)}{(2 \kappa+1)^{1 / 2}}$ & $-\frac{\sin \frac{\pi}{2}\left(\kappa-\frac{2}{3}\right)}{(2 \kappa+1)^{1 / 2}}$ \\
\hline 1 & $e^{-6 \pi i / 6} \sin \frac{\pi}{2}\left(\kappa-\frac{2}{3}\right)$ & $i \sin \frac{\pi}{2}\left(\kappa+\frac{2}{3}\right)$ & $\frac{e^{\pi i / 6} \cos \frac{\pi}{2}\left(\kappa-\frac{2}{3}\right)}{(2 \kappa+1)^{1 / 2}}$ & $-\frac{i \cos \frac{\pi}{2}\left(\kappa+\frac{2}{3}\right)}{(2 \kappa+1)^{1 / 2}}$ \\
\hline
\end{tabular}

7. The asymptotic forms of the Hermite functions. From the relation (4) between the equations (1) and (3), it is evident that the former equation has an even and an odd solution, and it is desirable to choose these in such a fashion that they reduce to the Hermite polynomials (2) when $\kappa$ is a positive integer. This result is achieved if these solutions, here denoted as $U_{k j}(z)$, be chosen so that

(35) $U_{\kappa 1}=(-1)^{\kappa / 2} \frac{\Gamma(\kappa+1)}{\Gamma\left(\frac{\kappa}{2}+1\right)} e^{z^{2} / 2} w_{1}, U_{\kappa 2}=2(-1)^{(\kappa-1) / 2} \frac{\Gamma(\kappa+1)}{\Gamma\left(\frac{\kappa+1}{2}\right)} e^{z^{2} / 2} w_{2}$, 
the first of which reduces to the polynomials of even powers when $\kappa$ is an even integer, the second to the polynomials of odd powers when $\kappa$ is odd. The following theorem is then evident:

THEOREM VII. The asymptotic forms of the solutions, $U_{k j}(z)$, of the equation (1), which at $z=0$ have the values

$$
\begin{aligned}
& U_{\kappa 1}(0)=(-1)^{\kappa / 2} \frac{\Gamma(\kappa+1)}{\Gamma\left(\frac{\kappa}{2}+1\right)}, U_{k 1}^{\prime}(0)=0, \\
& U_{k 2}(0)=0, \quad U_{\kappa 2}^{\prime}(0)=2(-1)^{(\kappa-1) / 2} \frac{\Gamma(\kappa+1)}{\Gamma\left(\frac{\kappa+1}{2}\right)},
\end{aligned}
$$

are described by equations (35) in terms of the asymptotic forms of $w_{j}(z)$, the latter, with their regions of validity, being given in Theorems I to VI.

By restricting $\kappa$ to positive integral values, the asymptotic forms of the Hermite polynomials (2) are obtained from (35). The simplification is obvious, except in the case of the coefficients of formula $(21)$, [ $\sin (\pi \kappa / 2)$ ], $\kappa$ even, and $[\cos (\pi \kappa / 2)], \kappa$ odd, the question being whether these reduce to quantities [0], or vanish entirely. That the latter is the case becomes evident by noting, from (35), that $w_{j} \rightarrow 0$ as $x \rightarrow \infty$, but $e^{i \xi} \rightarrow 0$ and $e^{-i \xi} \rightarrow \infty$ as $x \rightarrow \infty$; hence, if the formula (21) for $w_{j}$ is valid, the coefficients $[\sin (\pi \kappa / 2)]$ and $[\cos (\pi \kappa / 2)]$ must vanish when $\kappa$ is respectively an even and an odd integer. With this fact, and the familiar asymptotic formula for the gamma function,

$$
\Gamma(\kappa+1) \sim \kappa^{\kappa} e^{-\kappa}(2 \pi \kappa)^{1 / 2}[1],
$$

the following theorem is deduced from Theorem VII:

THEOREM VIII. The asymptotic forms of the Hermite polynomials (2) are given by the formulas

$$
\begin{aligned}
& \text { (a) } U_{k}(z) \sim \frac{2^{\kappa / 2} e^{\left(z^{2}+\kappa\right) / 2} \kappa 1}{\pi^{1 / 2} \kappa^{(\kappa+1) / 2}\left(1-\frac{z^{2}}{2 \kappa+1}\right)^{1 / 4}} \\
& \quad \times\left\{\cos \left(\frac{\pi \kappa}{2}-z(2 \kappa+1)^{1 / 2}\right)+O\left(\kappa^{-1 / 2}\right)\right\}, \\
& \text { (b) } U_{k}(z) \sim \frac{2^{\kappa / 2-1} e^{\kappa+z^{2}-z\left(z^{2}-2 \kappa-1\right)^{1 / 2} / 2} \kappa !}{\pi^{1 / 2} \kappa^{(\kappa+1) / 2}\left(\frac{z^{2}}{2 \kappa+1}-1\right)^{1 / 4}}
\end{aligned}
$$




$$
\times\left\{\frac{z}{(2 \kappa+1)^{1 / 2}}+\left(\frac{z^{2}}{2 \kappa+1}-1\right)^{1 / 2}\right\}^{\alpha+1 / 2}[1]
$$

(c) $U_{k}(z)=\frac{e^{z^{2} / 2} \kappa !}{(2 \kappa+1)^{1 / 3}}\left(\frac{2 e}{\kappa}\right)^{\kappa / 2}\left(\frac{\Phi}{3 \phi}\right)^{1 / 2}$

$$
\times\left\{e^{\pi i / 6} J_{-1 / 3}(\xi)+e^{5 \pi i / 6} J_{1 / 3}(\xi)+O\left(\kappa^{-1}\right)\right\},
$$

(d) $U_{\kappa}(z)=\frac{e^{z^{2} / 2}(2 e)^{\kappa / 2} \kappa !}{\pi^{1 / 2} \kappa^{\kappa / 2}(2 z)^{2 / 3} 3^{1 / 6}}$

$$
\times\left\{\Gamma\left(\frac{1}{3}\right)-\frac{6^{1 / 3} \Gamma\left(\frac{2}{3}\right)}{2} \eta+\frac{\Gamma\left(\frac{1}{3}\right)}{24} \eta^{3}+\cdots+O\left(\kappa^{-1}\right)\right\}
$$

valid in the half plane $R(z) \geqq 0$ as follows:

(a) inside the semi-circle $|z|=(2 \kappa+1)^{1 / 2}$;

(b) in the regions $\Xi^{(1)}$ and $\Xi^{(0)}$ as shown in figure $3(\mathrm{c})$;

(c) in a region of dimension $O\left(\kappa^{-1 / 6}\right)$ about the point $z=(2 \kappa+1)^{1 / 2}$;

(d) for values of $z$ in the neighborhood of the point $z=(2 \kappa+1)^{1 / 2}$.

The approximations (30) may be used in (c), and the variable $\eta$ of (d) is defined by (31).

8. Comparison with known formulas. In the article to which reference has been made, ${ }^{*}$ Watson determined the asymptotic forms of two independent solutions of the equation (3), $D_{-x-1}( \pm i z)$, these formulas being

$$
D_{-\kappa-1}( \pm i z)=\frac{\pi e^{z^{2} / 2}\left(\frac{\kappa}{e}\right)^{\kappa / 2}}{\Gamma(\kappa+1)} e^{\mp i z(2 \kappa)^{1 / 2}}\left\{e^{-z^{2} / 2}+O\left(\kappa^{-1 / 2}\right)\right\}
$$

in which $z$ is subject to the restriction,

$$
|y|<\frac{2^{1 / 2} \kappa^{\alpha}}{6}, 0 \leqq \alpha<\frac{1}{2},
$$

and $x$ is finite. The solutions are ordinarily represented by the power series

$$
D_{-\kappa-1}( \pm i z) \doteq e^{z^{2 / 2}} \sum_{n=0}^{\infty} \frac{\Gamma\left(\frac{m+\kappa+1}{2}\right)}{m ! \Gamma(\kappa+1)}(\mp 2 i z)^{m} 2^{(\kappa-1) / 2}
$$

which are uniformly convergent in the finite part of the plane. Although Watson gave no bound for $x$ in terms of $\kappa$, it is evident, because of the finiteness of $x$ and the unboundedness of $\kappa$, that the values $z$ may assume in (37) are included in the set of values $z$ may take on in the formulas (27).

\footnotetext{
* Cf. Introduction, p. 340.
} 
The solutions $w_{j}$ are expressible by usual means in terms of $D_{-x-1}( \pm i z)$, since the values of the latter and their derivatives at the origin are known from the series expansions (38). The computations show that

$$
\begin{aligned}
& w_{1}(z)=\frac{2^{-(\kappa+1) / 2} \Gamma(\kappa+1)}{\Gamma\left(\frac{\kappa+1}{2}\right)}\left\{D_{-\kappa-1}(i z)+D_{-\kappa-1}(-i z)\right\}, \\
& w_{2}(z)=\frac{i 2^{-(\kappa+3) / 2} \Gamma(\kappa+1)}{\Gamma\left(\frac{\kappa}{2}+1\right)}\left\{D_{-\kappa-1}(i z)-D_{-\kappa-1}(-i z)\right\},
\end{aligned}
$$

and the substitution of the forms (37) into the equations (39), with the use of the asymptotic formula for the gamma function, leads to the forms

$$
\begin{aligned}
& w_{1}(z) \sim\left\{\begin{array}{l}
\cos z(2 \kappa)^{1 / 2}+O\left(\kappa^{-1 / 2}\right), 0 \leqq|y| \leqq \epsilon, \\
\cos z(2 \kappa)^{1 / 2}\left(1+O\left(\kappa^{-1 / 2}\right)\right),|y|>\epsilon,
\end{array}\right. \\
& w_{2}(z) \sim(2 \kappa)^{-1 / 2}\left\{\begin{array}{l}
\sin z(2 \kappa)^{1 / 2}+O\left(\kappa^{-1 / 2}\right), 0 \leqq|y| \leqq \epsilon, \\
\sin z(2 \kappa)^{1 / 2}\left(1+O\left(\kappa^{-1 / 2}\right)\right),|y|>\epsilon,
\end{array}\right.
\end{aligned}
$$

which are asymptotically equivalent to the formulas (27) when $\kappa$ is large.

Adamoff's results for the Hermite polynomials on the finite part of the real axis are, of course, included in Watson's work and need not be considered separately. Plancherel and Rotach's results, however, take in the entire real axis and need separate consideration. Since they do not give a formula comparable to (36c), only the remaining formulas of (36) for $z=x$ need to be considered.

For the range of values $0 \leqq x<(2 \kappa+2)^{1 / 2}$, they give the formula

$$
U_{\kappa}(x) \sim \frac{e^{\left(x^{2}+\kappa+1\right) / 2} \kappa ! 2^{\kappa / 2}}{\pi^{1 / 2}(\kappa+1)^{(\kappa+1) / 2}\left(1-\frac{x^{2}}{2 \kappa+2}\right)^{1 / 4}}\left\{\cos \left(\frac{\pi \kappa}{2}-x(2 \kappa+2)^{1 / 2}\right)+O\left(\kappa^{-1 / 2}\right)\right\},
$$

which is asymptotically equivalent to (36a) since

$$
(\kappa+1)^{(\kappa+1) / 2}=\kappa^{(x+1) / 2} e^{1 / 2}[1] .
$$

For $x>(2 \kappa+2)^{1 / 2}$, the formula given by them may be reduced, by obvious simplifications, to the form

$$
\frac{2^{\kappa / 2-1} \kappa ! \exp \left(\frac{\kappa+x^{2}-x\left(x^{2}-2 \kappa-2\right)^{1 / 2}}{2}\right)}{\pi^{1 / 2} \kappa^{(\kappa+1) / 2}\left(\frac{x^{2}}{2 \kappa+2}-1\right)^{1 / 4}}
$$




$$
\times\left(\frac{x}{(2 \kappa+2)^{1 / 2}}+\left\{\frac{x^{2}}{2 \kappa+2}-1\right\}^{1 / 2}\right)^{\kappa+1 / 2}\left[1+O\left(\kappa^{-21 / 2}\right)\right]
$$

the ratio of which to (36b) is asymptotic to the quantity

$$
\exp \left(-\frac{x}{2}\left\{\left(x^{2}-2 \kappa-2\right)^{1 / 2}-\left(x^{2}-2 \kappa-1\right)^{1 / 2}\right\}\right)\left(\frac{2 \kappa+1}{2 \kappa+2}\right)^{(2 \kappa+1) / 4} ;
$$

the exponential in this expression is asymptotic to exp ( $\left.\frac{1}{4}\right)$, and the second factor is asymptotic to exp $\left(-\frac{1}{4}\right)$, so that the expression itself is asymptotic to unity. Hence the two forms under comparison are asymptotically equivalent.

In the neighborhood of the point $x=(2 \kappa+2)^{1 / 2}$, Plancherel and Rotach determined the asymptotic form of the Hermite polynomials as a power series

$$
\frac{2^{\kappa-2 / 3} \kappa ! e^{3 x^{2} / 4}}{\pi 3^{1 / 6} x^{\kappa+2 / 3}}\left\{\Gamma\left(\frac{1}{3}\right)+\frac{6^{1 / 3} \Gamma\left(\frac{2}{3}\right)}{2^{4 / 3}} t-\frac{\Gamma\left(\frac{1}{3}\right)}{6} t^{3}+\cdots+O\left(\kappa^{-1 / 3}\right)\right\},
$$

in which

$$
t=\frac{2 \kappa+2-x^{2}}{(2 x)^{2 / 3}}
$$

and, with a little manipulation, this series can be shown asymptotically equivalent to (37d). Now near $x=(2 \kappa+1)^{1 / 2}, x-(2 \kappa+1)^{1 / 2}=O\left(\kappa^{-1 / 6}\right)$, so that

$$
(2 \kappa)^{1 / 2}=x\left(1+\frac{E_{1}}{\kappa^{2 / 3}}\right)
$$

in which $E_{1}$ is a bounded function of $\kappa$. Hence

but, since

$$
\begin{aligned}
e^{\kappa / 2} & =\exp \left(\frac{x^{2}}{4}+E_{1} \kappa^{1 / 3}\right)\left[1+O\left(\kappa^{-1 / 3}\right)\right], \\
(2 \kappa)^{\kappa / 2} & =x^{k}\left(1+\frac{E_{1}}{\kappa^{2 / 3}}\right)^{\kappa}
\end{aligned}
$$

$$
\left(1+\frac{E}{n}\right)^{n}=e^{E}[1]
$$

when $n$ is large, it follows that

$$
\left(1+\frac{E_{1}}{\kappa^{2 / 3}}\right)^{\kappa}=\left\{\left(1+\frac{E_{1}}{\kappa^{2 / 3}}\right)^{\kappa / 8}\right\}^{\kappa^{1 / 3}}=\exp \left\{E_{1 \kappa^{1 / 8}}\left(1+O\left(\kappa^{-2 / 3}\right)\right)\right\}
$$

or 


$$
\left(1+\frac{E_{1}}{\kappa^{2 / 3}}\right)^{\kappa}=\exp \left(E_{1} \kappa^{1 / 3}\right)\left[1+O\left(\kappa^{-1 / 3}\right)\right]
$$

From these facts it is evident that

and since

$$
\frac{e^{\kappa / 2}}{\kappa^{\kappa / 2}}=\frac{e^{x^{2} / 42^{\kappa / 2}}}{x^{\kappa}}\left[1+O\left(\kappa^{-1 / 3}\right)\right]
$$

$$
\eta=-2^{2 / 3} t+O\left(\kappa^{-1 / 3}\right),
$$

it is seen that the series (40) is equivalent asymptotically to the series (36d).

UNIVERSITY OF Wisconsin, Madison, Wis. 